\title{
A New Radiation Correction Method for Remote Sensing Images Based on Change Detection
}

\author{
Juan Wang, Xijia Liu, XiaoMing Tao ${ }^{(\bowtie)}$, and Ning Ge \\ Tsinghua National Laboratory for Information Science and Technology (TNList), \\ Tsinghua University, Beijing, People's Republic of China \\ taoxm@tsinghua.edu.cn
}

\begin{abstract}
As an important remote sensing image pre-processing method, radiation correction is essential to reduce deviation introduced by environment factors, especially for tasks such as image compression, image fusion, and object recognition. In this paper, we propose a new radiation correction method for remote sensing images based on change detection. Due to the fact that areas of with significant changes deteriorate performance of radiation correction, these sections should be detected and discarded in the image firstly. Then general radiation correction technology is considered to have better performance on the rest sections. The core idea of the proposed method exists in the combination of radiation correction and change detection. Experimental results prove validness of the proposed method. As an application example, this method used in image compression shows better performance than other compression technologies.
\end{abstract}

Keywords: Radiation correction $\cdot$ Remote sensing image $\cdot$ Change detection . Histogram matching

\section{Introduction}

Remote sensing images are widely used in many fields, such as crop cultivation, weather prediction, resource investigation, military strategy and so on, creating tremendous ecological, social, scientific and military benefits. However, raw remote sensing images are hardly put into service when obtained from the satellites in most cases. Kinds of factors can contribute to the unsatisfactory image quality, including inter-structure of sensor, thermal noise from chips, weather condition, shadows form clouds and mist and the angle of the sun. Radiation correction technology is a common means specialized in eliminating external negative influences and improving visual effects. Moreover, radiation correction plays an important part in image fusion technology to reconcile two images, also does work in object recognition technology to remove the differences of irrelevant objects and highlight the target object.

Traditional radiation correction methods contain histogram matching and linear fitting as representatives for simple methods, as well as complex methods such as solution of radiation transmission equation, analysis of ground spectral test data and multiband comparison [1]. Regretfully, complex methods seldom come into practice 
because of difficulty in data collection, huge amounts of computation and complicated operations. Of course, simple methods always can't accomplish expected results due to inherent simplicity. In this paper, we represent a new radiation correction method for remote sensing images based on change detection. The proposed method hands over two tasks to traditional histogram matching technology. Firstly, iterative histogram matching is used to detect remarkable changes. Secondly, histogram matching is only used to correct radiative errors in rest plain areas. The combination of change detection and radiation correction can efficiently make up the disadvantages of simple radiation correction methods.

The remainder of the paper is organized as follows. In Sect. 2, we will review the histogram matching in detail. Then we introduce the new radiation correction method based on change detection in Sect. 3. In Sect. 4, experimental results are presented. Finally, we conclude the proposed method and conceive the future work in Sect. 5.

\section{Preliminary Technology}

\subsection{Histogram}

Histogram represents the pixel number of every grayscale value, reflecting the distribution of grayscale value. Histogram is a fundamental statistics characteristic of an image, as well as the essential of histogram matching technology.

Let $r_{k}$ denote the grayscale value, $n_{k}$ denote the pixel number of $r_{k}$, and $n$ denote total pixel numbers of an image. The probability $p_{r}\left(r_{k}\right)$ is defined as follows:

$$
\begin{aligned}
p_{r}\left(r_{k}\right) & =n_{k} / n \\
0 \leq r_{k} \leq 1 \quad k & =0,1,2, \ldots, l-1
\end{aligned}
$$

The relationship between $r_{k}$ and $p\left(r_{k}\right)$ depicted in rectangular coordinate system is what we called histogram.

\subsection{Histogram Transformation}

Grayscale transformation function $T$ is given as follows:

$$
s=T(r)
$$

After transformation, every previous grayscale value $r$ will correspond to a new grayscale value $s$.

Grayscale transformation function $T$ should demand conditions as follows:

(a) $T(r)$ should increase monotonously when $0 \leq r \leq 1$;

(b) $0 \leq T(r) \leq 1$ when $0 \leq r \leq 1$. 
Let $p_{s}(s)$ represent the probability of new grayscale value $s$ transformed from $r$, then we have:

$$
p_{s}(s)=p_{r}(r) \cdot \frac{d}{d s}\left[T^{-1}(s)\right]=\left[p_{r}(r) \cdot \frac{d r}{d s}\right]_{r=T^{-1}(s)}
$$

In (3), it's clear that transformation function $T$ can adjust the distribution of grayscale by controlling the probability density function, which is the theoretical fundament of histogram matching.

\subsection{Histogram Matching}

The main idea of histogram matching [2] is to make the histogram of an image similar to the histogram of another image. By modifying the histogram, both the interested area can be highlighted and image quality can be improved.

Let $\left\{r_{k}\right\}$ denote the grayscale of original image, $\left\{z_{k}\right\}$ denote the grayscale of target image with expected histogram. The goal is to find a transformation function $H$ satisfying the condition as follows:

$$
z=H(r)
$$

Algorithm:

(a) Regard accumulation distribution function as transformation function:

$$
\begin{aligned}
& s=T(r)=\int_{0}^{r} p_{r}(\omega) d \omega \\
& v=G(z)=\int_{0}^{z} p_{z}(\omega) d \omega
\end{aligned}
$$

(b) Inverse transformation function $G$ :

$$
z=G^{-1}(v)
$$

(c) Let $s$ replace $v$ :

$$
z=G^{-1}(s)
$$

(d) Obtain a compound transformation function:

$$
z=G^{-1}(T(r))=G^{-1} T(r)
$$

So the expected transformation function $H$ is as follows:

$$
H=G^{-1} T
$$




\section{The Proposed Method}

Our radiation correction method is in fact an improved histogram matching. Generally, histogram matching only functions as a means to make two images approximate. Plain images can get excellent results while images with abundant texture, such as remote sensing images, often perform poorly. The reasons both root in the simple transformation operation of histogram matching and changeable details in remote sensing images.

In order to find a simple and efficient way to correct radiation errors of remote sensing images, we creatively regard histogram matching as a change detection mean [3]. The general flows are shown in Fig. 1. First, histogram matching is iteratively applied to reference image, then areas which have worst matching results will be removed from the image in every iteration. That is to say, histogram matching in every iteration aims to get better matching results for the next time by discarding unwanted areas. In most cases, these areas just correspond to the most obvious changes, so we call this process as change detection.

It's indispensable to mention that these changed areas need to be ex-tended to regular rectangles firstly and only the maximum rectangle will be removed then. The main purpose is to prevent too many areas from removal. Besides, rectangular boundary is helpful to remain the rest areas regular, also convenient for transmission if necessary.

After several iterations, the rest areas are plainer than before. At present, histogram matching will serve as a radiation correction means to the rest areas, good performance can be expected.

Then it's necessary to discuss the impact of these 'discarded' areas. As we mentioned before, their existence is likely to cause the failure of radiation correction to some extents. However, if they are removed from the image, superior correction results in the rest areas can be guaranteed at least. Moreover, sometimes it doesn't matter to neglect a few areas. For example, in image fusion technology, the effect of radiation correction makes more sense in plain background areas than changed objects. Overall, in some specific application fields, sacrificing these areas for better radiation correction performance of rest areas is reasonable.

Performance can be evaluated by comparing standard deviation. Standard deviation is defined as follows:

$$
\sigma=\sqrt{\frac{1}{N} \sum_{k=1}^{N}\left(I_{t}(k)-I_{h}(k)\right)^{2}}
$$

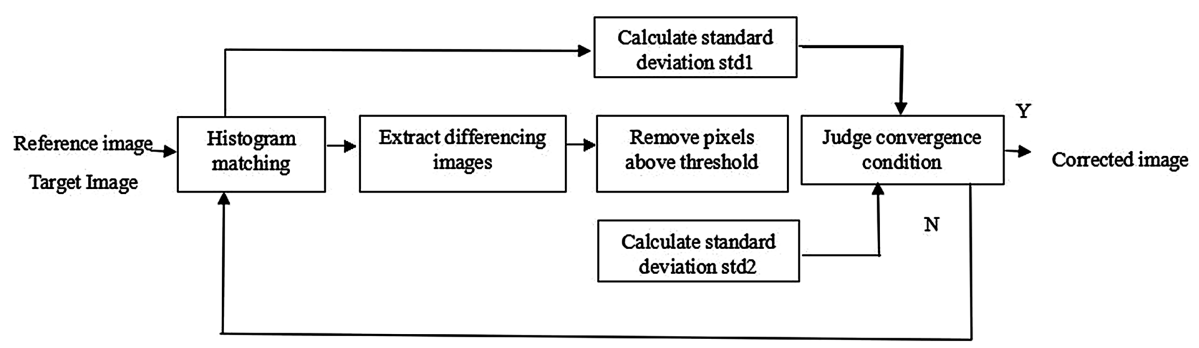

Fig. 1. Block diagram of the proposed method 
Where $N$ denotes pixel numbers in the rest areas, $I_{t}(k)$ and $I_{h}(k)$ denote the grayscale value of target image and corrected image respectively. It's easy to understand that standard deviation is lower, radiation correction performs better.

The detailed algorithm is presented as follows:

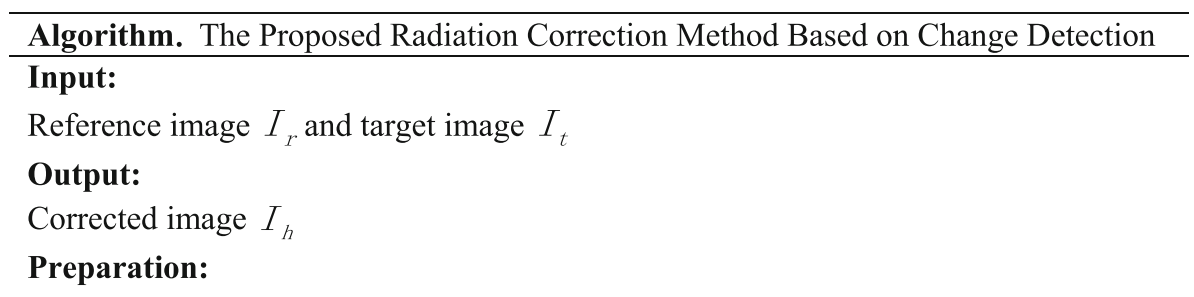

1. Matrix $L$ and $\mathrm{B}$ have the same size as $I_{r}$, recording the location of removed pixels, initial elements in $L$ and $\mathrm{B}$ are all 1;

2. Apply histogram matching to $I_{r}$ and denote the histogram-matched image as $I_{h}(i)$;

3. Extract differencing image $I_{d}(i)=\left|I_{t}(i)-I_{h}(i)\right|$

4. Calculate standard deviation of differencing image $\sigma(i)=\operatorname{std}\left(I_{d}(i)\right)$;

\section{Repeat:}

5. Set a proper threshold $T$;

6. Scan differencing image and compare every pixel value $I_{d}(x, y)$ with $T$;

7. If $\left|I_{d}(x, y)\right|>T$, then $\operatorname{set} L(x, y)=0$;

8. Connect the areas where $L(x, y)=0$ by 8 connected domain;

9. Surround every region by minimum rectangle;

10. Choose the maximum rectangle, let $B(x, y)=0$ where $(x, y)$ belongs to the rectangle;

11. $I_{r}(x, y)$ and $I_{t}(x, y)$ are removed from $I_{r}$ and $I_{t}$ respectively where $B(x, y)=0$;

12. Set $\mathrm{i}=\mathrm{i}+1$;

13.Apply histogram matching to the non-removal areas where $B(x, y)=1$ as $I_{h}(i)$;

14.Extract differencing image $I_{d}(i)=\left|I_{t}(i)-I_{h}(i)\right|$ in the areas where $B(x, y)=1$;

15. Calculate standard deviation of differencing image.

$$
\sigma(i)=\operatorname{std}\left(I_{d}(i)\right)
$$

Until:

$|\sigma(i)-\sigma(i-1)|<\varepsilon$

\section{Return}




\section{Results and Discussions}

\subsection{Simulation on Remote Sensing Images}

To confirm the effectiveness of the proposed method, we choose 10 reference images and 10 target images respectively from remote sensing image datasets. Making the reference image get close to the target image as much as possible is supposed.

Figure 2 shows one pair of the tested images. As we can see, although the scene is the same, radiation errors lead to many gray differences. There is a hypothesis that distortion is imposed on reference image because of natural factors, what we need to do is setting target image as standard to eliminate distortion of reference image.

If traditional histogram matching without change detection is applied on the reference image, the result is illustrated in Fig. 3. Figure 3(a) shows histogram-matched

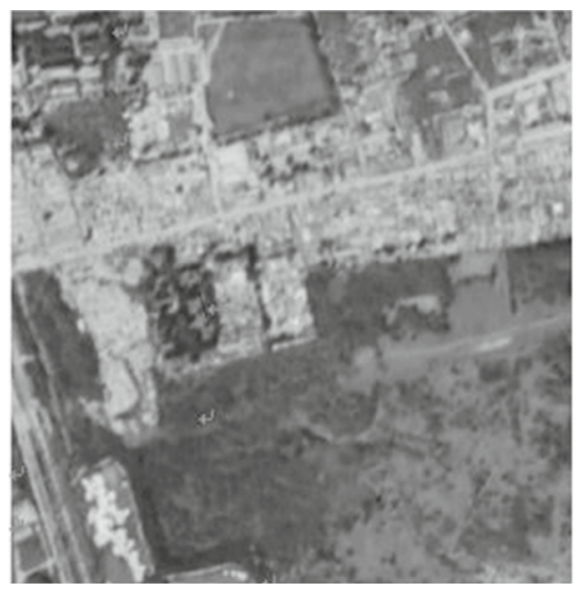

(a) Reference image

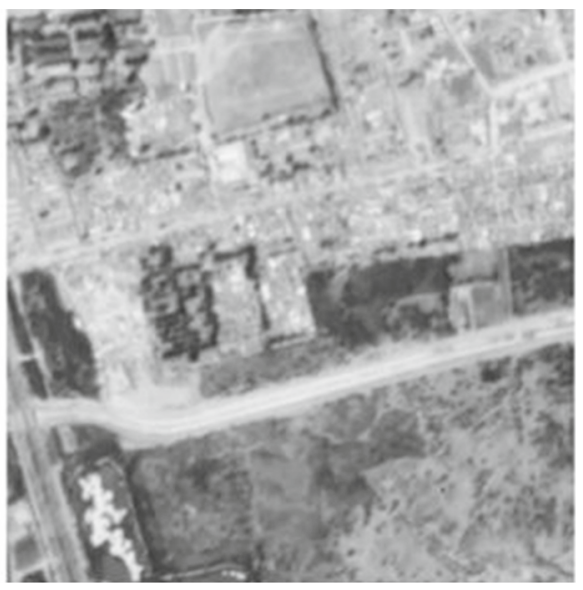

(b) Target image

Fig. 2. Test images

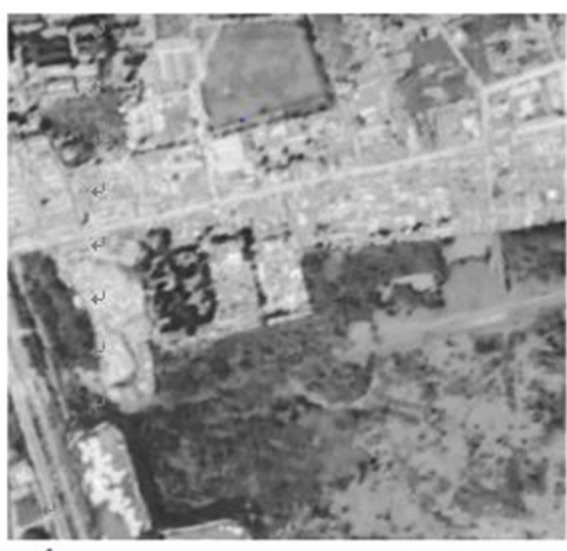

(a) Histogram-matched image without

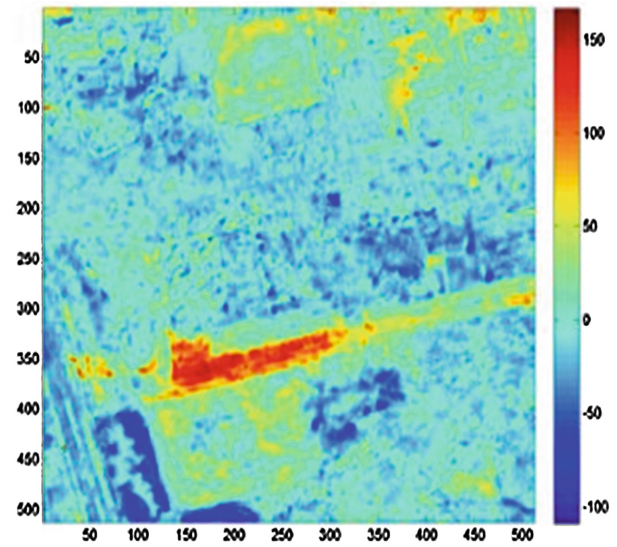

(b) Differencing image

change detection

Fig. 3. Ordinary histogram mathching without change detection 
image, but observing the image directly is difficult to evaluate the performance, so we also show the differencing image extracted from target image and histogram-matched image in (b). Light blue areas represent perfect histogram matching performance while red and dark blue areas are opposite. Pitifully, some areas in the differencing image take on unexpected color. More specifically, standard deviation of the differencing image is 30.56 .

By contrast, we use our method on the same pair images. The primary step is to detect changes. From Fig. 3(b), the most remarkable difference is the red area located in the middle of the image. So in the first iteration, this area is extended into rectangle and removed, shown in Fig. 4(a). As Fig. 4(b) - (d) illustrate, in the following iterations, other obvious differences are removed in the same way. As a result, the difference image is gradually covered by larger and larger light blue areas, and the standard

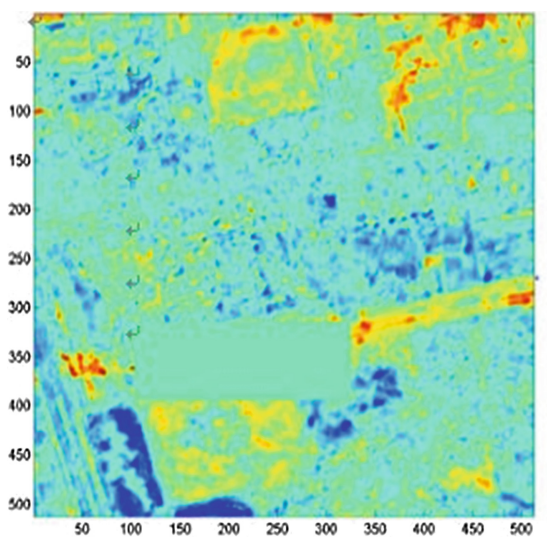

(a) 1 st differencing image

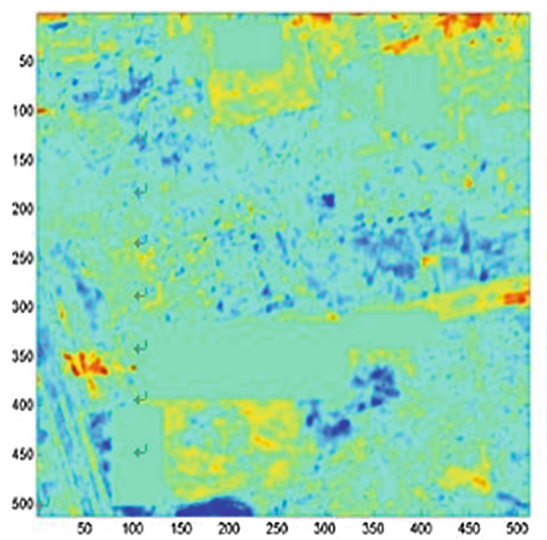

(c) 5th differencing image

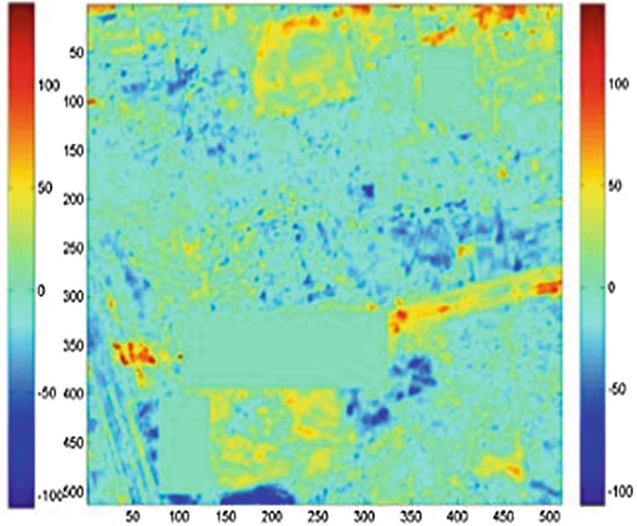

(b) 3th differencing image

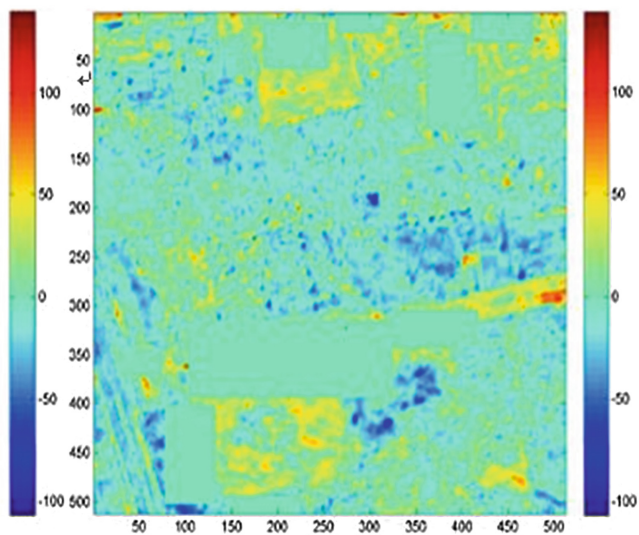

(d) 9th differencing image

Fig. 4. The process of the proposed method 
deviation drops from 30.56 to 20.07 , indicating effective improvement of radiation correction performance.

\subsection{Parameters Impact on Results}

Similar to [3], there is no restriction on the threshold. However, if $T$ is too large, few changes are removed every time so that too many iterations will be carried on to converge. On the contrary, if $T$ is too small, it will be a high price to remove huge parts from the image. In order to make the threshold adaptive to the change of image, $T$ depends on the new differencing image. Experiments show that $5 \%$ of pixels removed from the differencing image is reasonable, as observed from Fig. 5. When it comes to $\varepsilon$, it decides when the iterations should stop. In fact, $\varepsilon$ and $T$ are complementary,

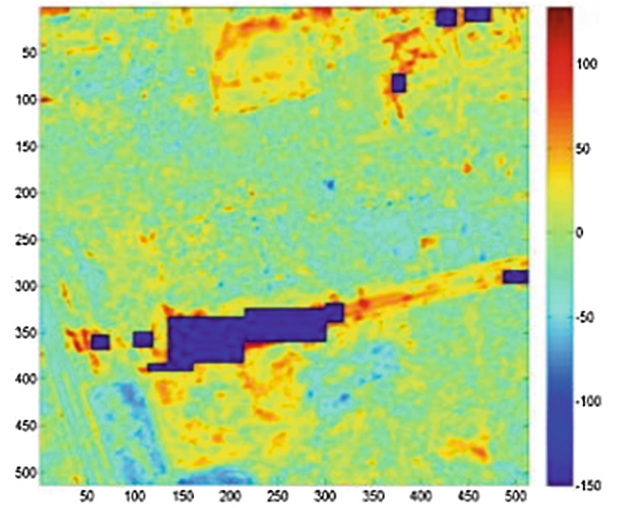

(a) $1 \%$ pixels removed

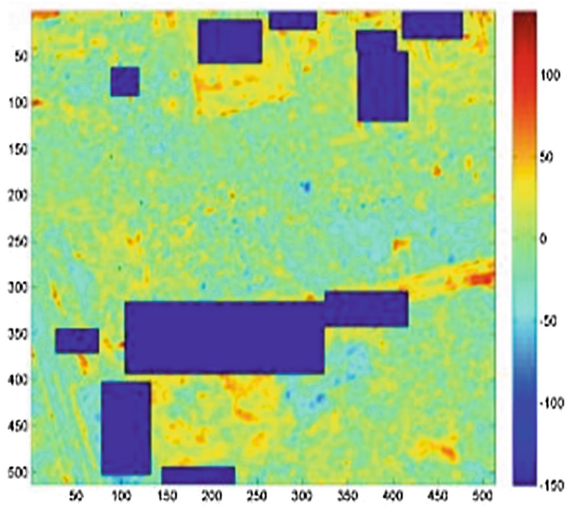

(b) $5 \%$ pixels removed

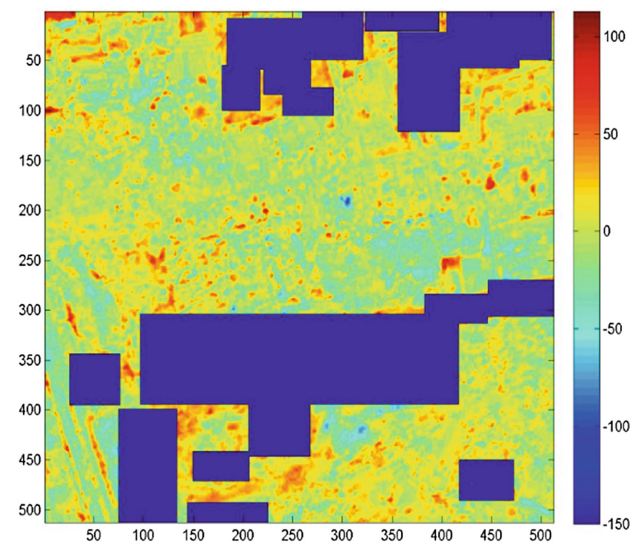

(c) $10 \%$ pixels removed

Fig. 5. The effect of threshold 


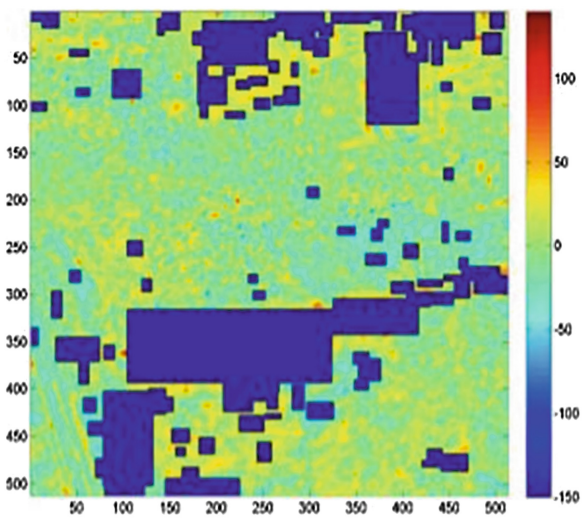

(a) $\mathcal{E}=0.01$

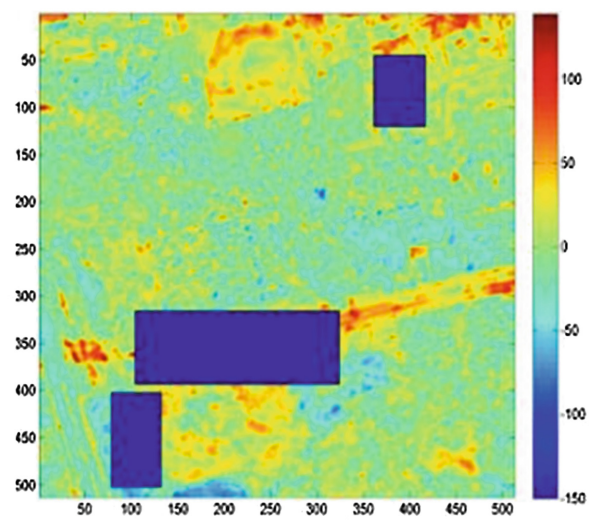

(b) $\mathcal{E}=0.1$

Fig. 6. The effect of $\varepsilon$

influencing converge condition jointly. Corresponding to $T$ discussed above, setting $\varepsilon$ as 0.05 is considered appropriate. Figure 6 illustrates the effect of different $\varepsilon$.

\subsection{Performance Comparison}

Table 1 shows standard deviation comparison of traditional histogram matching, linear fitting and proposed method. It's clear that our proposed method performs best in most cases. Of course, when calculating standard deviation of the proposed method, removed pixels are not included so that the comparison results may be nonsense sometimes. We have to mention that our proposed method is suitable for application scenes where some areas can be sacrificed to compensate other areas, such as in image compression field.

Table 1. Standard deviation comparison

\begin{tabular}{l|l|l|l}
\hline Image number & Traditional histogram matching & Linear fitting & Proposed method \\
\hline 1 & 16.5461 & 16.2601 & $\mathbf{1 5 . 3 0 5 7}$ \\
\hline 2 & 19.2085 & 18.5839 & $\mathbf{1 6 . 7 4 8 8}$ \\
\hline 3 & 31.7864 & 30.2404 & $\mathbf{1 9 . 0 3 0 9}$ \\
\hline 4 & 20.8124 & 20.4158 & $\mathbf{1 7 . 8 0 0 0}$ \\
\hline 5 & 16.5021 & 16.3427 & $\mathbf{1 6 . 2 3 8 7}$ \\
\hline 6 & 14.5939 & $\mathbf{1 4 . 5 0 4 8}$ & 14.5623 \\
\hline 7 & 13.9015 & $\mathbf{1 3 . 8 0 7 3}$ & 13.8808 \\
\hline 9 & 18.2179 & 17.6366 & $\mathbf{1 4 . 8 1 6 7}$ \\
\hline 10 & 13.4672 & 13.4898 & $\mathbf{1 3 . 3 9 9 3}$ \\
\hline & 15.1075 & 15.9477 & $\mathbf{1 5 . 0 2 4 7}$ \\
\hline
\end{tabular}




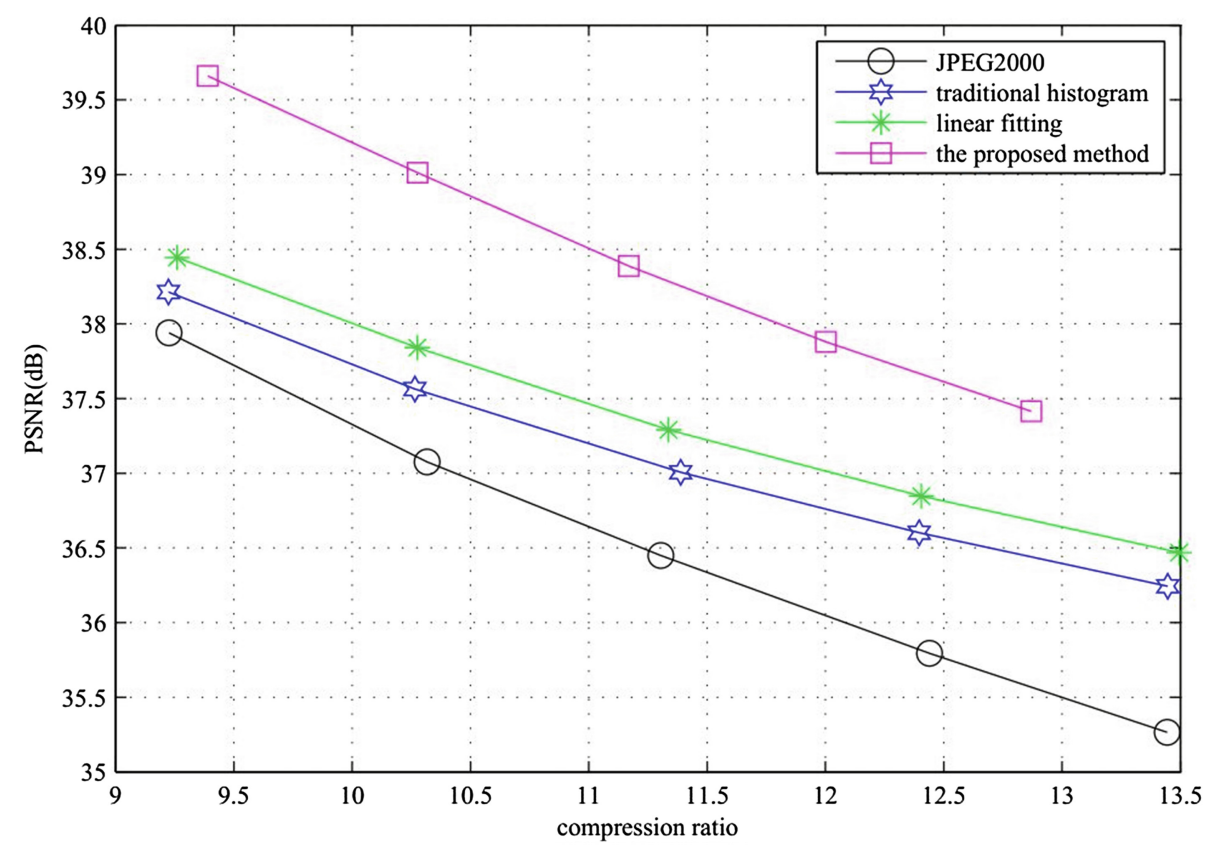

Fig. 7. The proposed method applied in image compression compared with other methods

\subsection{Application in Image Compression}

A concrete application example that some new remote sensing images from the satellite need to be transmitted to the ground. Similar previous images equipped both on the satellite and on the ground can be used to correct grayscale differences (radiation errors) by our method. We expect that corrected images can get very close to the new images. Then only differencing image and removed areas need to be transmitted, which is supposed to save much transmitted data compared with transmitting the whole new images. In order to reconstruct the new images, the receiver need to get the same corrected images in the same way, then add received differencing images and fill the removed areas in. We apply the proposed method, traditional histogram matching and linear fitting respectively to correct previous remote sensing images, then only the differencing images extracted from new images and corrected images are transmitted (the proposed method also includes the removed areas). Three radiation correction methods are compared with the art-of-state image compression standard JPEG2000 in Fig. 7. As we can see, remote sensing images compression indeed gets higher PSNR based on our proposed method than other methods. 


\section{Conclusions}

The proposed method efficiently improves the performance of radiation correction by adding change detection technology in. Traditional histogram matching technology is modified in this method, both used as a change detection means as well as a radiation correction means. Areas of with significant changes will be removed by iterative histogram matching firstly, on account of which, the following histogram matching is considered to have better performance on the rest areas. Not only the proposed method inherits the advantage of simplicity, but also experimental results prove its superiority to other general radiation correction methods. As an example, we represent how the proposed method is applied in remote sensing images compression. Simulation results show that there are about $1 \mathrm{~dB}$ and $2 \mathrm{~dB}$ reconstruction performance gains using the proposed method compared with the other two radiation correction methods and JPEG2000.

Acknowledgement. This work is supported by the National Basic Research project of China (973) (2013CB329006), National Natural Science Foundation of China NSFC, (No. 61471220, No. 61021001, No. 61132002), and Tsinghua University Initiative Scientific Research Program.

\section{References}

1. Wang, Y., Liu, A., Tao, S.: CBERS-2CCDradiation correction technology on remote sensing images. J. Telecommun. Technol. 48(4), 44-48 (2008). doi:10.3969/j.issn.1001-893X.2008. 04.010

2. Gonzalez, R.C.: Digital Image Processing. Pearson Education India, Noida (2004)

3. Yang, Z., Zhang, W., Wang, W., et al.: Change detection based on iterative invariant area histogram matching. In: International Conference on Geoinformatics, pp. 1-6. IEEE (2011)

4. Lu, D., Mausel, P., Brondízio, E., et al.: Change detection techniques. Int. J. Remote Sens. 25 (12), 2365-2401 (2004)

5. Zheng, L., Li, Z., Zhang, J.: Color matching of colorful remote sensing image. Mapp. Inf. Eng. 29(3), 29-31 (2004). doi:10.3969/j.issn.1007-3817.2004.03.012

6. Xu, H.: Landsat research and comparison on the remote sensing image normalization processing model. Earth Inf. Sci. 10(3), 294-301 (2008). doi:10.3969/j.issn.1560-8999.2008.03. 004 